\title{
EDITORIAL
}

\section{Neutrophilic inflammation: "Don't you go to pieces on me!"}

\author{
R.A. Stockley
}

$\mathbf{N}$ eutrophilic infiltration is a phenomenon observed even in the healthy lung. However, increased infiltration occurs in many acute and chronic lung diseases and although necessary as part of the lung's secondary defence systems, infiltration has been implicated in pathogenic processes leading to the tissue damage associated with many diseases. Not only do the neutrophil contents possess the ability to damage tissues, they may also generate chemotactic factors with the potential to amplify leukocyte infiltration [13].

There is a general acceptance that neutrophil recruitment, although necessary, is a direct cause of many of the features of acute and chronic lung disease, largely as a result of collateral damage caused by the release of oxidants and proteinases, as indicated above. This process has been implicated in acute respiratory distress syndrome [4] resulting in damage to alveolar cells and in several of the features of chronic obstructive pulmonary disease (COPD), including interstitial damage resulting in emphysema and airway damage resulting in loss of mucociliary function, excess mucus production and impairment of several other factors required for host defence [5].

Although extensive lung damage can follow acute pneumonias, this is not often the case and usually (despite severe neutrophilic inflammation) there can be clear resolution of the process with minimal or no apparent lung damage. This raises the very important question: why doesn't (rather than why should) neutrophil inflammation damage the lung? Pneumonia itself is undoubtedly an intense neutrophil inflammation but is associated with marked serum exudation and sometimes haemorrhage. This in its own right would bring in circulating antioxidant molecules and proteinase inhibitors, limiting damage by these molecules and proteins.

However, in recent years, many studies have investigated mechanisms of resolution of neutrophilic inflammation. Once the process that initiates neutrophilic recruitment is over, mechanisms of neutrophil removal need to take place. If cells die, the initiation of necrosis results in the cessation of metabolic processes that produce oxidants but can result in the spilling of the remaining proteinases that have been stored within the primary granules, thereby enhancing the potential of damage via these molecules. The process of apoptosis allows

CORRESPONDENCE: R.A. Stockley, Queen Elizabeth Hospital, Edgbaston, Birmingham B15 2TH UK. Fax: 44 1216978256. E-mail: r.a.stockley@bham.ac.uk neutrophils to "go quietly". Many studies have confirmed that this is a key process in neutrophil removal [6]. The cell undergoes nuclear and cytoplasmic changes and the senescent cells are recognised by macrophages and phagocytosed without activation [7]. This was thought to be a critical mechanism, at least in part explaining why severe neutrophilic inflammation in pneumonia may not result in any permanent damage.

This removal process raises the issue as to how neutrophils and particularly their proteinases can actually cause damage. Studies have suggested that very high concentrations of proteinases are released in the local environment of activated cells and have to diffuse away for some distance before the concentration approaches that of local inhibitors, resulting in enzyme inactivation [8]. Clearly this process takes place before neutrophils undergo apoptotic change. Eventually, the cells are no longer required and have to be removed from the lung either by the mucociliary escalator or by apoptosis. Studies have identified apoptotic cells in sputum samples [9] and in lung lavage (where they are also present in macrophages), indicating a quiescent removal process [10].

This latter process of ingestion is impaired by cigarette smoke [11], although the macrophages may also have a reduced ability to phagocytose apoptotic cells [12]. This, together with the reduced mucociliary clearance seen in COPD, would result in longer retention of apoptotic neutrophils. The fate of these apoptotic cells has, however, remained unknown, although more recent studies have identified the process of secondary necrosis. Indeed, if apoptotic cells are not cleared by normal mechanisms and undergo secondary necrosis, not only can they cause more extensive damage due to proteinase release but this may also lead to the perpetuation of the inflammatory process as a result of the local action of the enzymes.

In the current issue of the European Respiratory Journal, RYDELLTÖRMÄNEN et al. [13] look in detail at the fate of neutrophils in mice over time. Using the installation of endotoxin, the authors were able to generate focal areas of inflammation within the lung, enabling the time course to be studied in more detail. The paper identified early infiltration, showing that cells are adopting a relatively quiescent phenotype in the airway wall. Once in the lumen they appear as a more activated phenotype, and later, apoptotic changes are identified together with significant necrosis. The time of course suggests that early infiltration and activation occurs within $4 \mathrm{~h}$, but by $24 \mathrm{~h}$ apoptosis and cell debris are identified and secondary necrosis is clearly evident at $36 \mathrm{~h}$ (suggesting the apoptotic clearance process is overwhelmed), although neutrophils persist even at 
$72 \mathrm{~h}$ after this stimulating challenge, suggesting a perpetuation of inflammatory response.

These observations raise the possibility that many of the pathological changes seen in COPD and, indeed, the persistence of the inflammation once smoking has ceased [14] may reflect a balance between the apoptotic clearance mechanisms of neutrophils and the development of subsequent secondary necrosis. If the inflammation in response to cigarette smoke leads to impaired phagocytosis of apoptotic cells, the result would be reduced clearance of cells enabling secondary necrosis to take place, which would not only cause further damage but perpetuate the inflammatory process by amplifying it through proteinase activity. Once smoking ceases, the increased inflammation in the airways may continue to exceed the phagocytic potential for apoptotic cells, and the impaired mucociliary clearance may result in these cells being retained and hence undergoing secondary necrosis. The net result would be continued inflammation in the airway.

It would be of interest to use the animal model reported in the current issue [13] and assess how this model is influenced in the smoking mouse to determine whether an additive effect disturbing the balance between apoptosis and necrosis occurs, thereby providing further insight into the relevance of chronic obstructive pulmonary disease. Furthermore, studies of bacterial or viral infections assessing the balance of apoptotic clearance versus secondary necrosis may provide a clearer understanding of how exacerbation of chronic obstructive pulmonary disease may lead to subsequent lung function decline. RYDELL-TÖRMÄNEN et al. [13] further our knowledge of the potential destructive nature of neutrophilic inflammation and hence generate understanding of the nature of future protective therapeutic strategies.

\section{REFERENCES}

1 Henson PM, Johnston RB. Tissue injury in inflammation. Oxidants, proteinases and cationic proteins. J Clin Invest 1987; 79: 669-674.

2 Malech HD, Gallin JL. Neutrophils in human diseases. N Engl J Med 1988; 317: 687-694.

3 Weiss SJ. Tissue destruction by neutrophils. $N$ Engl J Med 1989; 320: 365-375.

4 Griffiths MJD, Evans TW. Acute respiratory distress syndrome. In: Gibson GJ, Geddes DM, Costabell U, Sterk
PJ, Corrin B, eds. Respiratory Medicine. London, Elsevier Science Ltd, 2003; pp. 736-763.

5 Sullivan AL, Stockley RA. Proteinases in COPD. In: Hansell TT, Barnes PJ, Burkhausser V, eds. Recent Advances of Physiology of COPD. Basel, Birkhäuser, 2004; pp. 75-99.

6 Haslett C. Granulocyte apoptosis and its role in the resolution and control of lung inflammation. Am J Respir Crit Care Med 1999; 160: S5-S11.

7 Savill JS, Wyllie AH, Henson JE, Walport MJ, Henson PM, Haslett C. Macrophage phagocytosis and aging neutrophils in inflammation. Programme cell death in the neutrophil leads to recognition by macrophages. J Clin Invest 1989; 83: 865-875.

8 Liou TJ, Campbell EJ. Quantum proteolysis resulting from release of single granules by human neutrophil: a novel non-oxidative mechanism of extracellular proteolytic activity. J Immunol 1996; 157: 2624-2631.

9 Erjefalt JS, Larsson S, Persson CGA, Nihlen U, Lofdahl CG, Greiff L. Necrosis of neutrophils and eosinophils together with granulocyte-derived granule proteins in directly fixed sputum samples from COPD patients. Am J Respir Crit Care Med 2002; 168: A598.

10 Cox G, Crossley J, Xing Z. Macrophage engulfment of apoptotic neutrophils contributes to the resolution of acute pulmonary inflammation in vivo. Am J Respir Cell Mol Biol 1995; 12: 232-237.

11 Wickenden JA, Clarke MC, Rossi AG, et al. Cigarette smoke prevents apoptosis through inhibition of caspase activation and induced necrosis. Am J Respir Cell Mol Biol 2003; 29: 562-570.

12 Hodge S, Hodge G, Scicchitano R, Reynolds PN, Holmes M. Alveolar macrophages from subjects with chronic obstructive pulmonary disease are deficient in their ability to phagocytose apoptotic airway epithelial cells. Immunol Cell Biol 2003; 81: 289-296.

13 Rydell-Törmänen K, Uller L, Erjefalt JS. Direct evidence of secondary necrosis of neutrophils during intense lung inflammation. Eur Respir J 2006; 28: 268-274.

14 Willemse BWM, ten Hacken NHT, Rutgers B, LesmanLeegte IGAT, Postma DS, Timens W. Effect of 1-year smoking cessation on airway inflammation in COPD and asymptomatic smokers. Eur Respir J 2005; 26: 835-845. 